\title{
GC-MS ANALYSIS OF METHANOLIC STEM EXTRACT OF GYNOCHTHODES RIDSDALEI, RAZAFIM AND B. BREMER, AN ENDEMIC, ENDANGERED MEDICINAL PLANT OF SOUTHERN WESTERN GHATS
}

\author{
RENJI R. NAIR, A. GANGAPRASAD* \\ Plant Tissue Culture and Molecular Biology Lab, Department of Botany, University of Kerala, Thiruvananthapuram, Kerala, India, 695581 \\ Email: agangaprasad@yahoo.com
}

Received: 27 Dec 2016, Revised and Accepted: 27 Mar 2017

\section{ABSTRACT}

Objective: The present research study was undertaken to determine the presence of bioactive components present in the methanolic stem extract of Gynochthodes ridsdalei using Gas Chromatography-Mass Spectrometry (GC-MS) analysis.

Methods: the Fresh stem of Gynochthodes ridsdalei collected from the forest areas of Ponmudi region of Thiruvananthapuram district of Kerala state, India was used. The mass spectrum GC-MS of the crude methanolic extract was estimated using the database of National Institute of Standard and Technology (NIST).

Results: The active principles with their retention time, peak area, molecular formula, molecular weight, structure and category of the compound were predicted. The analysis revealed the presence of 52 bioactive components. Most of the identified compounds are basically biological important. The components were identified by comparing their retention time and peak area with that of literature and by interpretation of mass spectra. The phyto components screened were of biological importance. Some of them were sterols, anthraquinones, vitamins etc.

Conclusion: The result reveals the existence of various bioactive compounds and validates the earlier reports of therapeutic importance of the plant. Gynochthodes ridsdalei is recommended as a plant of phytochemical and pharmaceutical importance.

Keywords: Gynochthodes ridsdalei, Morinda reticulata, endangered, southern Western Ghats, gas chromatography

(C) 2017 The Authors. Published by Innovare Academic Sciences Pvt Ltd. This is an open access article under the CC BY license (http://creativecommons.org/licenses/by/4.0/) DOI: http://dx.doi.org/10.22159/ijcpr.2017v9i3.19665

\section{INTRODUCTION}

The use of medicinal plants has gained considerable importance in our day to day life since ancient times. Traditional medicine is the sum total of knowledge, skills and practices based on the theories, beliefs, experiences indigenous to different cultures that are used to maintain health as well as to diagnose, improve or treat physical and mental illness. The therapeutic use of some plants against critical human illnesses predates recorded history and represents the most significant direct antecedent to modern medicine [1]. Medicinal plants are rich resources of ingredients which can be used in drug development and synthesis. Many higher plants are a major source of secondary metabolites which are used for many medicinal purposes. Gynochthodes ridsdalei (Syn: Morinda reticulata) is a large woody climbing shrub with coriaceous reticulate leaves belonging to the family Rubiaceae. The plant is endemic to southern Western Ghats [2]. It forms an important component in a variety of herbal formulation in traditional medicine [3]. Plants belonging to family Rubiaceae are known to contain a substantial amount of anthraquinones especially in the roots [4] and are characterised by brightly coloured anthraquinones that have been used in the past for various dyeing purpose. The screening of plant extracts is an innovative method to find therapeutically important compounds which will help to develop novel drugs [5]. Gas Chromatography-Mass Spectrometry (GC-MS) analysis is used for the direct analysis of bioactive components in traditional medicine and for separation and analysis of multicomponent mixtures such as essential oils, hydrocarbons etc [6].

\section{MATERIALS AND METHODS}

\section{Plant material}

Fresh stem of Gynochthodes ridsdalei collected from the forest areas of Ponmudi region of Thiruvananthapuram district of Kerala state, India was used. The taxonomical identification of the plant was done using authentic literature $[7,8]$. A voucher specimen was deposited at the Herbarium of Department of Botany, University of Kerala, Kariavattom (KUBH No. 8095).

\section{Preparation of plant extract}

The collected stem was chopped and shade dried under room temperature for $7 \mathrm{~d}$ and then milled into coarse powder by the mechanical grinder. About 10 gm of the powdered stem sample was subjected to Soxhlet extraction using $200 \mathrm{ml}$ methanol. The extract was concentrated using rotary evaporator (Superfit rotavap) under reduced pressure and stored in the refrigerator until further use. Two microliters of the extract were employed in GC-MS analysis for analysis of different compounds.

\section{GC-MS analysis}

The analysis of the extract was performed using GC-MS (Model: GC MS-QP 2010, Shimadzu, Tokyo, Japan) equipped with a VF $5 \mathrm{~ms}$ fused silica capillary column of $30 \mathrm{~m}$ length, $0.25 \mathrm{~mm}$ diameter and $0.25 \mu \mathrm{m}$ film thickness. For GC-MS detection, electron ionization energy of $70 \mathrm{eV}$ was used. The carrier gas was helium (99.9\%) and used at constant flow rate of $1.2 \mathrm{ml} / \mathrm{min}$. Injector and mass transfer line temperature were set at $200^{\circ} \mathrm{C}$ and $255^{\circ} \mathrm{C}$ respectively. The oven temperature was set from 70 to $300^{\circ} \mathrm{C}$ at $10^{\circ} \mathrm{C} / \mathrm{min}$ for $9 \mathrm{~min}$. One microliter of the sample was injected in a split mode with a scan range of $40-1000 \mathrm{~m} / \mathrm{z}$. The total running time of GC-MS was $35 \mathrm{~min}$. The relative percentage amount of each component was calculated by comparing its average peak area normalization [9].

\section{Identification of the components}

Elucidation of mass spectrum GC-MS was conducted using the database of National Institute Standard and Technology (NIST) and Wiley Spectra Libraries. The spectrum of the unknown component was compared with the spectrum of known components, which was stored in the NIST library source [10]. The name, molecular weight and molecular mass of the identified compounds were further confirmed by comparison of their retention indices with literature data. For quantitative analysis, compounds concentrations (as \% content) were calculated by integrating their corresponding chromatographic peak area. 


\section{RESULTS AND DISCUSSION}

The bioactive components present in the methanolic stem extract of $G$ ridsdalei were identified by GC-MS analysis. The gas chromatogram shows the relative concentrations of various compounds getting eluted as a function of retention time (fig. 1). Identification of the compounds was accomplished by comparing their mass spectra and retention indices with those given in the literature and those authentic samples. The active principles with their retention time (RT), molecular formula, molecular weight (MW), concentration (\%), nature of the compound and their biological activities are presented in (table 1) and are listed by their order of retention times. The heights of the peak indicate the relative concentrations of the compounds present in $G$ ridsdalei.

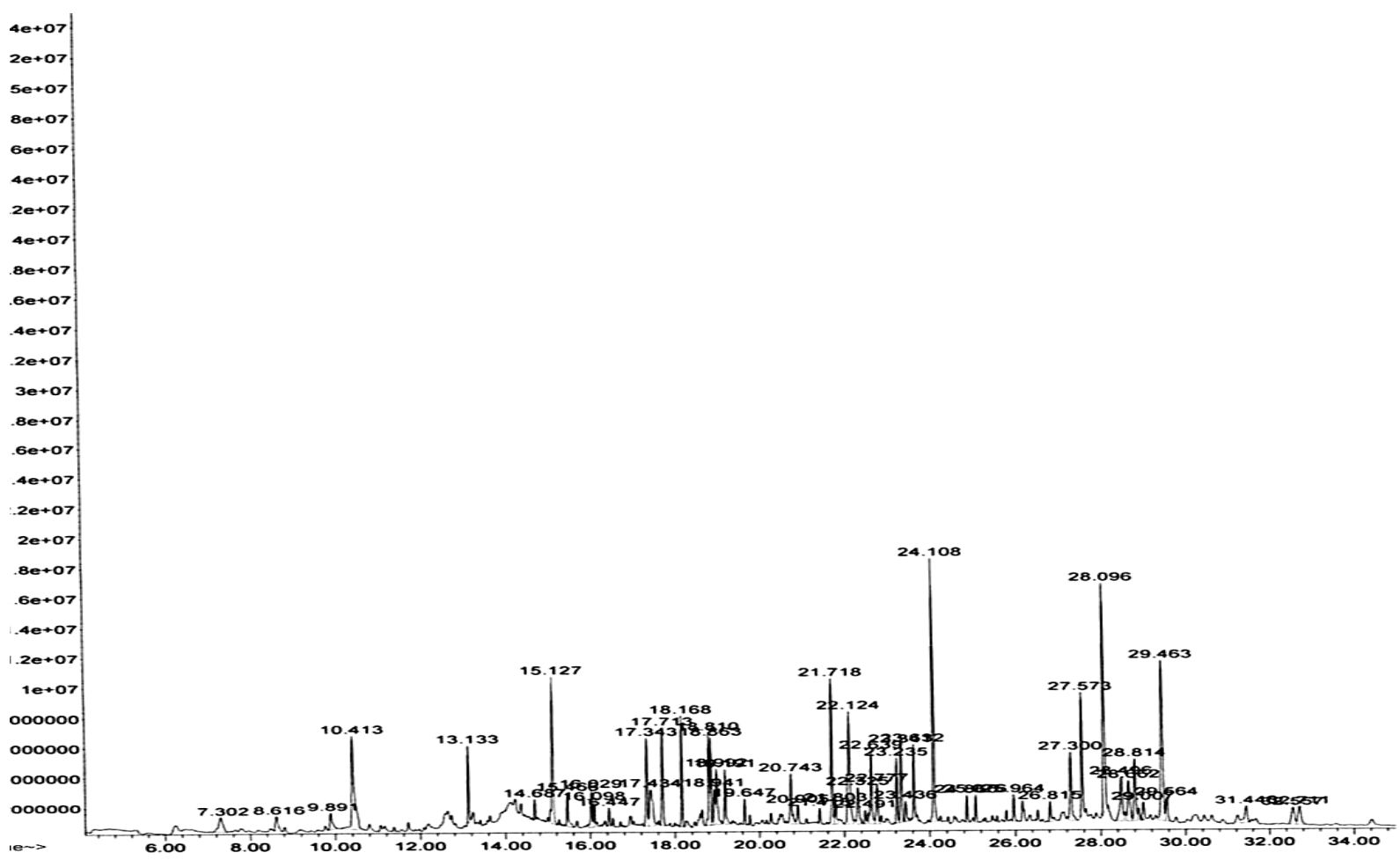

Fig. 1: GC-MS Chromatogram of methanolic stem extract of Gynochthodes ridsdalei

Table 1: Phytocomponents identified in the methanolic stem extract of G. ridsdalei by GC-MS

\begin{tabular}{|c|c|c|c|c|c|c|c|}
\hline $\begin{array}{l}\text { S. } \\
\text { No. }\end{array}$ & $\begin{array}{l}\text { Retention } \\
\text { time }\end{array}$ & $\begin{array}{l}\text { Peak } \\
\text { area\% }\end{array}$ & Name of the compound & $\begin{array}{l}\text { Molecular } \\
\text { formula }\end{array}$ & $\begin{array}{l}\text { Molecular } \\
\text { weight }\end{array}$ & $\begin{array}{l}\text { Nature of } \\
\text { compound }\end{array}$ & Uses \\
\hline 1 & 7.303 & 1.16 & 1,3-Benzenediol, 5-chloro- & $\mathrm{C}_{6} \mathrm{H}_{8} \mathrm{O}_{4}$ & 144.1253 & $\begin{array}{l}\text { Phenol } \\
\text { (Resorcinol) }\end{array}$ & $\begin{array}{l}\text { Diazodyes, } \\
\text { Dermatology }\end{array}$ \\
\hline 2 & 8.618 & 0.83 & $\begin{array}{l}\text { 5-Hydroxymethylfurfura } \\
\text { Benzene methanol, 3-fluoro- }\end{array}$ & $\mathrm{C}_{6} \mathrm{H}_{6} \mathrm{O}_{3}$ & 126.1100 & Organic compound & Baking industry \\
\hline 3 & 9.889 & 0.80 & $\begin{array}{l}\text { 2-Methoxy-4-vinylphenol } \\
\text { 3-Methoxyacetophenone }\end{array}$ & $\mathrm{C}_{7} \mathrm{H}_{7} \mathrm{NO}_{4}$ & 169.136 & Phenol & Flavoring agent \\
\hline 4 & 10.416 & 5.09 & $\begin{array}{l}\text { Phenol, 2,6-dimethoxy- } \\
\text { 3-Amino-2,6-dimethoxypyridine }\end{array}$ & $\mathrm{C}_{8} \mathrm{H}_{10} \mathrm{O}_{3}$ & 154.1632 & $\begin{array}{l}\text { Syringol } \\
\text { Phenol }\end{array}$ & $\begin{array}{l}\text { Smoky aroma in } \\
\text { foods }\end{array}$ \\
\hline 5 & 13.135 & 1.60 & 1,Butanol, 3-methyl, formate & $\mathrm{C}_{6} \mathrm{H}_{12} \mathrm{O}_{2}$ & 116 & $\begin{array}{l}\text { Alcoholic } \\
\text { compound }\end{array}$ & Antimicrobial \\
\hline 6 & 14.688 & 0.39 & $\begin{array}{l}\text { Phenol, 2,6-dimethoxy-4-(2- } \\
\text { propeny D-3-Hydroxy-4- } \\
\text { methoxycinnamic acid }\end{array}$ & $\mathrm{C}_{11} \mathrm{H}_{14} \mathrm{O}_{3}$ & 194.230 & $\begin{array}{l}\text { Phenol } \\
\text { Eugenol }\end{array}$ & $\begin{array}{l}\text { Perfumary } \\
\text { dentistry }\end{array}$ \\
\hline 7 & 15.126 & 4.34 & $\begin{array}{l}\text { 4-((IE)-3-Hydroxy-l-propenyl)-2- } \\
\text { me thoxyphenol }\end{array}$ & $\mathrm{C}_{10} \mathrm{H}_{22} \mathrm{O}_{3}$ & $\mathrm{C}_{10} \mathrm{H}_{12} \mathrm{O}_{3}$ & Coniferyl alcohol & $\begin{array}{l}\text { Fungal growth } \\
\text { inhibitor }\end{array}$ \\
\hline 8 & 15.468 & 1.00 & $\begin{array}{l}\text { 3,5-Dimethoxy-4- } \\
\text { hydroxyphenylacetic acid }\end{array}$ & С8H803 & 152.147 & Acid & Synthesis of atenolol \\
\hline 9 & 16.032 & 1.02 & 2,5-Diethoxyaniline & $\mathrm{C}_{6} \mathrm{H}_{11} \mathrm{~N}$ & 121.18 & Aniline & $\begin{array}{l}\text { Precursor to crystal } \\
\text { violet dye }\end{array}$ \\
\hline 10 & 16.099 & 0.56 & 6-Octen-l-ol & $\mathrm{C}_{10} \mathrm{H}_{20} \mathrm{O}$ & 156.27 & $\begin{array}{l}\text { Citronellol } \\
\text { (monoterpenoid) }\end{array}$ & $\begin{array}{l}\text { Insect repellents, } \\
\text { perfumary }\end{array}$ \\
\hline 11 & 16.448 & 0.42 & 3-Methyl-l-penten-4-yn-3-ol & $\mathrm{C}_{6} \mathrm{H}_{10} \mathrm{O}$ & 80.128 & Tertiary hexanol & Hypnotic/sedative \\
\hline 12 & 17.340 & 2.58 & n-Hexadecanoic acid & $\mathrm{C}_{16} \mathrm{H}_{32} \mathrm{O}_{2}$ & 256.4241 & Palmitic acid & $\begin{array}{l}\text { Antioxidant } \\
\text { hypocholesterolemic } \\
\text {, nematicide }\end{array}$ \\
\hline 13 & 17.436 & 2.29 & Scopoletin & $\mathrm{C}_{10} \mathrm{H}_{8} \mathrm{O}_{4}$ & 192.16 & Coumarin & Used in food making \\
\hline 14 & 17.711 & 2.69 & Squalene & $\mathrm{C}_{30} \mathrm{H}_{50}$ & 410 & Triterpene & Antimicrobial, \\
\hline
\end{tabular}




\begin{tabular}{|c|c|c|c|c|c|c|c|}
\hline & & & & & & & $\begin{array}{l}\text { antioxidant, } \\
\text { antitumour }\end{array}$ \\
\hline 15 & 18.172 & 2.24 & 1-Butanol,3-Methyl, Formate & $\mathrm{C}_{6} \mathrm{H}_{12} \mathrm{O}_{2}$ & 116 & Alcoholic & Antimicrobial \\
\hline 16 & 18.810 & 2.35 & 9,10-Anthracenedione, & $\mathrm{C}_{14} \mathrm{H}_{8} \mathrm{O}_{2}$ & 208.22 & Anthraquinone & Dyes \\
\hline 17 & 18.862 & 1.98 & Phytol & $\stackrel{\bullet}{{ }_{20} \mathrm{H}_{40} \mathrm{O}}$ & 296 & Diterpene & $\begin{array}{l}\text { Anticancer, } \\
\text { antimicrobial, } \\
\text { anti-inflammatory }\end{array}$ \\
\hline 18 & 18.944 & 1.01 & 9,12-Octadecadienoic acid (Z,Z)- & $\mathrm{C}_{18} \mathrm{H}_{32} \mathrm{O}_{2}$ & 280.4455 & $\begin{array}{l}\text { Linoleic acid } \\
\text { (carboxylic acid) }\end{array}$ & $\begin{array}{l}\text { Antioxidant effect, } \\
\text { paints, varnishes }\end{array}$ \\
\hline 19 & 18.989 & 1.72 & $\begin{array}{l}\text { Oleic Acid cis-13-Octadecenoic } \\
\text { acid cis-Vaccenic acid }\end{array}$ & $\mathrm{C}_{18} \mathrm{H}_{34} \mathrm{O}_{2}$ & 282.4614 & Fatty acid & $\begin{array}{l}\text { Reducing blood } \\
\text { pressure }\end{array}$ \\
\hline 20 & 19.189 & 1.48 & Octadecanoic acid & $\mathrm{C}_{18} \mathrm{H}_{36} \mathrm{O}_{2}$ & 284.4772 & Stearic acid & Dietary supplements \\
\hline 12 & 19.650 & 0.57 & $\begin{array}{l}\text { 1-Hydroxy-2- } \\
\text { methylanthraquinone } \\
\text { l-Hydroxy-4- } \\
\text { methylanthraquinone }\end{array}$ & $\mathrm{C}_{15} \mathrm{H}_{10} \mathrm{O}_{3}$ & 238.242 & Anthraquinone & $\begin{array}{l}\text { Dyes, Medicinal } \\
\text { Importance }\end{array}$ \\
\hline 22 & 20.742 & 1.76 & $\begin{array}{l}\text { 9,10-Anthracenedione, } 2 \text { - } \\
\text { hydroxy-l-methoxy }\end{array}$ & $\mathrm{C}_{19} \mathrm{H}_{18} \mathrm{O}_{7}$ & 358.342 & Anthraquinone & $\begin{array}{l}\text { Dyes, Medicinal } \\
\text { importance }\end{array}$ \\
\hline 23 & 20.905 & 0.64 & 9-Octadecenamide, (Z)- & $\mathrm{C}_{18} \mathrm{H}_{35} \mathrm{NO}$ & 281.4766 & Amide & $\begin{array}{l}\text { For depression, } \\
\text { sleep disorders }\end{array}$ \\
\hline 24 & 21.418 & 0.40 & $\begin{array}{l}\text { 1,2,4-Benzenetricarboxylic acid, } \\
\text { 5-methyl-, trimethyl ester- }\end{array}$ & $\mathrm{C}_{12} \mathrm{H}_{12} \mathrm{O}_{6}$ & 252.2201 & Trimellitic acid & Unknown \\
\hline 25 & 21.715 & 4.03 & $\begin{array}{l}\text { 2-(Hydroxymethyl) } \\
\text { anthraquinones } \\
\text { 1,4,7-Trimethyl-2-azafluorene } \\
\text { 4-Propylxanthen-9-one }\end{array}$ & $\mathrm{C}_{15} \mathrm{H}_{10} \mathrm{O}_{3}$ & 238.238 & Anthraquinone & $\begin{array}{l}\text { Dyes, Medicinal } \\
\text { importance }\end{array}$ \\
\hline 26 & 21.804 & 0.65 & $\begin{array}{l}\text { 9,10-Anthracenedione, } 1,5- \\
\text { dimethoxybenzopyrenol }\end{array}$ & $\mathrm{C}_{14} \mathrm{H}_{10} \mathrm{~N}_{2} \mathrm{O}_{2}$ & 238.241 & Anthraquinone & $\begin{array}{l}\text { Dyes, Medicinal } \\
\text { importance }\end{array}$ \\
\hline 27 & 22.124 & 4.42 & $\begin{array}{l}\text { 1-Hydroxy-4- } \\
\text { methylanthraquinone } \\
\text { 3-Phenoxy-2H-chromen-2-one } \\
\text { 2,6-Diaminoanthraquinone }\end{array}$ & $\mathrm{C}_{15} \mathrm{H}_{10} \mathrm{O}_{3}$ & 238.242 & Anthraquinone & $\begin{array}{l}\text { Dyes, Medicinal } \\
\text { importance }\end{array}$ \\
\hline 28 & 22.324 & 1.32 & $\begin{array}{l}\text { 1-Hydroxy-4- } \\
\text { methylanthraquinone } \\
\text { 9,10-Anthracenediol, 2-ethyl- }\end{array}$ & $\mathrm{C}_{15} \mathrm{H}_{10} \mathrm{O}_{3}$ & 238.242 & Anthraquinone & $\begin{array}{l}\text { Dyes, Medicinal } \\
\text { importance }\end{array}$ \\
\hline 29 & 22.488 & 0.35 & Docosanoic acid & $\mathrm{C}_{22} \mathrm{H}_{44} \mathrm{O}_{2}$ & 340.5836 & $\begin{array}{l}\text { Behenic acid) } \\
\text { carboxylic acid }\end{array}$ & $\begin{array}{l}\text { Detergents, floor } \\
\text { polishes }\end{array}$ \\
\hline 30 & 22.636 & 2.20 & $\begin{array}{l}\text { 9,10-Anthracenedione, l- } \\
\text { hydroxy-2-(hydroxymethyl)- }\end{array}$ & $\mathrm{C}_{15} \mathrm{H}_{10} \mathrm{O}_{4}$ & 254.238 & Anthraquinone & $\begin{array}{l}\text { Dyes, Medicinal } \\
\text { importance }\end{array}$ \\
\hline 31 & 22.777 & 1.31 & $\begin{array}{l}\text { 9,10- } \\
\text { Anthracenedione,1,8-dihydrox y- } \\
\text { 3-methyl- }\end{array}$ & $\mathrm{C}_{18} \mathrm{H}_{18} \mathrm{~N}_{2} \mathrm{O}_{4}$ & 326.346 & Anthraquinone & $\begin{array}{l}\text { Dyes, Medicinal } \\
\text { importance }\end{array}$ \\
\hline 32 & 23.238 & 1.44 & $\begin{array}{l}\text { 4-Ethenyl- } \\
\text { 2-methoxyphenol }\end{array}$ & $\mathrm{C}_{9} \mathrm{H}_{10} \mathrm{O}_{2}$ & 150.18 & $\begin{array}{l}\text { Aromatic } \\
\text { compound }\end{array}$ & Flavouring agent \\
\hline 33 & 23.342 & 2.09 & Benzoic acid, heptadecyl ester & $\mathrm{C}_{23} \mathrm{H}_{28} \mathrm{~N} \mathrm{O}_{3}$ & 380.48 & $\begin{array}{l}\text { Aromatic } \\
\text { carboxylic acid }\end{array}$ & Food preservative \\
\hline 34 & 23.439 & 0.59 & $\begin{array}{l}\text { Fumaric acid, cis-hex-3-enyl tetra } \\
\text { decyl Ester }\end{array}$ & $\mathrm{C}_{4} \mathrm{H}_{4} \mathrm{O}_{4}$ & 116.07 & $\begin{array}{l}\text { Unsaturated fatty } \\
\text { acid }\end{array}$ & Food industry \\
\hline 35 & 23.632 & 1.69 & $\begin{array}{l}\text { Octadecanoic acid, 2,3- } \\
\text { dihydroxypr opyl ester }\end{array}$ & $\mathrm{C}_{21} \mathrm{H}_{42} \mathrm{O}_{4}$ & 358.5558 & $\begin{array}{l}\text { Glycerol ester of } \\
\text { stearic acid }\end{array}$ & Food additive \\
\hline 36 & 24.107 & 7.31 & 13-Docosenamide, (Z)- & $\mathrm{C}_{22} \mathrm{H}_{43} \mathrm{NO}$ & 337.5829 & $\begin{array}{l}\text { Erucid acid (Fatty } \\
\text { acid) }\end{array}$ & $\begin{array}{l}\text { Lubricant, biodiesel } \\
\text { fuel precursor }\end{array}$ \\
\hline 37 & 24.865 & 0.62 & $\begin{array}{l}\text { 22-Tricosenoic acid } \\
\text { Triacontyl acetate }\end{array}$ & $\begin{array}{l}\mathrm{CH}_{3}\left(\mathrm{CH}_{2}\right)_{21} \\
\mathrm{COOH}\end{array}$ & 354.61 & Fatty acid & Oil paints, lubricants \\
\hline 38 & 25.073 & 0.67 & 4,5-Dibenzopyrene & $\mathrm{C}_{20} \mathrm{H}_{12}$ & 302.35 & $\begin{array}{l}\text { Aromatic } \\
\text { hydrocarbon }\end{array}$ & $\begin{array}{l}\text { Naturally emitted } \\
\text { coal tar }\end{array}$ \\
\hline 39 & 25.964 & 0.69 & $\begin{array}{l}\text { gamma.-Tocophero } \\
\text { beta.-Tocopherol }\end{array}$ & $\mathrm{C}_{2} 8 \mathrm{H}_{48} \mathrm{O}_{2}$ & 416.680 & Vitamin E & Antioxidant \\
\hline 41 & 27.301 & 2.49 & $\begin{array}{l}\text { Campesterol } \\
\text { 5-Cholestene-3-ol,24-methyl- }\end{array}$ & $\mathrm{C}_{28} \mathrm{H}_{48} \mathrm{O}$ & 400.69 & Phytosterol & Lowering cholesterol \\
\hline 42 & 27.569 & 3.46 & Stigmasterol & $\mathrm{C}_{29} \mathrm{H}_{48} \mathrm{O}$ & 412.6908 & $\begin{array}{l}\text { Unsaturatd } \\
\text { phytosterol }\end{array}$ & Food making \\
\hline 43 & 28.096 & 7.59 & $\begin{array}{l}\text { gamma.-Sitosterol } \\
\text { beta.-Sitosterol }\end{array}$ & $\mathrm{C} 29 \mathrm{H} 500$ & 414.71 & Sterol & $\begin{array}{l}\text { Lowers blood } \\
\text { cholesterol }\end{array}$ \\
\hline 44 & 28.497 & 2.22 & $\begin{array}{l}\text { Stigmast-7-en-3-ol, } \\
\text { Cholest-4-en-3-one } \\
\text { Pregn-4-ene-3, 20-dione, ( } 8 . \\
\text { alpha, 10. alpha.)- }\end{array}$ & $\mathrm{C}_{27} \mathrm{H}_{46} \mathrm{O}$ & 386.65 & Sterol & Food making \\
\hline 45 & 28.661 & 1.74 & Tetradecanoic acid & $\mathrm{C}_{14} \mathrm{H}_{28} \mathrm{O}_{2}$ & 228.37 & Fatty acid & $\begin{array}{l}\text { Antioxidant, Cancer } \\
\text { preventive }\end{array}$ \\
\hline 46 & 28.817 & 2.23 & $\begin{array}{l}\text { 4,22-Stigmastadiene-3-one } \\
\text { Spinasterone }\end{array}$ & $\mathrm{C}_{39} \mathrm{H}_{46} \mathrm{O}$ & 410.686 & Phytosterol & $\begin{array}{l}\text { Precursor of vitamin } \\
D_{3}\end{array}$ \\
\hline 47 & 29.010 & 0.65 & Cyclohex-2-enone, 2 & $\mathrm{C}_{6} \mathrm{H}_{8} \mathrm{O}$ & 96.13 & Ketone & $\begin{array}{l}\text { Synthesis of } \\
\text { pharmaceuticals and }\end{array}$ \\
\hline
\end{tabular}




\begin{tabular}{|c|c|c|c|c|c|c|c|}
\hline & & & & & & & fragrances \\
\hline 48 & 29.463 & 6.71 & Stigmast-5-en-3-one & $\mathrm{C}_{29} \mathrm{H}_{50} \mathrm{O}$ & 414.72 & Beta-Phytosterol & Food preparations \\
\hline 49 & 29.567 & 0.91 & Imidazolidine & $\mathrm{C}_{3} \mathrm{H}_{8} \mathrm{~N}_{2}$ & 72.109 & Diamine & Muscle relaxant \\
\hline 50 & 31.447 & 1.04 & Cholestan-3-one & $\mathrm{C}_{27} \mathrm{H}_{48}$ & 372.68 & Triterpene & Food industry \\
\hline 51 & 31.494 & 2.83 & Stigmasterol & $\mathrm{C}_{29} \mathrm{H}_{48} \mathrm{O}$ & 412 & Sterol & $\begin{array}{l}\text { Precursor of vitamin } \\
D_{3}\end{array}$ \\
\hline 52 & 32.561 & 1.00 & Gibbane-1,10-diarboxyli acid & $\mathrm{C}_{19} \mathrm{H}_{22} \mathrm{O}_{6}$ & 182.22 & Heterocylic acid & $\begin{array}{l}\text { Fungicides, making } \\
\text { dyes }\end{array}$ \\
\hline
\end{tabular}

The analysis revealed the presence of 52 photo components. Major compounds detected were sterols, anthraquinones, terpenes, vitamins etc. Sitosterol (7.59\%) showed highest peak (dominant component), followed by docos enamide. Among the identified compounds, the diterpene alcohol, phytol is vital in the dispensation of glucose and can trigger enzymes within the body that have strong positive effects on insulin level. This means that phytol in the human diet could perhaps help reinstate the metabolic activities of those with type- 2 diabetes $[11,12]$. It is also a constituent of chlorophyll in plants and precursor for the manufacture of synthetic forms of vitamin $E$ [13].

Stigmasterol is an unsaturated phytosterol occurring in the plant fats or oils. Stigmasterol is also found in various vegetables, legumes, nuts, seeds etc. Stigmasterol is used as a precursor in the manufacture of semisynthetic progesterone, a valuable human hormone that plays an important physiological role in the regulatory and tissue rebuilding mechanisms related to estrogen effects, as well as acting as an intermediate in the biosynthesis of androgens, estrogens, and corticoids [14]. It is also used as the precursor of vitamin $D_{3}$. Recently squalene possesses chemo preventive activity against colon carcinogenesis $[15,16]$.

\section{CONCLUSION}

This is the first report on the analysis of bioactive components present in $G$. ridsdalei. The result reveals the existence of various bioactive compounds and validates the earlier reports of therapeutic importance of the plant. G. ridsdalei is recommended as a plant of phytochemical and pharmaceutical importance. Further studies can be done to isolate the active principle of the methanolic extract as well as to elucidate the effect of extract for various diseases.

\section{ACKNOWLEDGEMENT}

The authors express their sincere gratitude to the Head, Department of Botany, University of Kerala for providing necessary facilities for doing this work and to the University of Kerala for financial support in the form of Junior Research Fellowship is acknowledged by the first author.

\section{CONFLICT OF INTERESTS}

Declare none

\section{REFERENCES}

1. Dutt R, Garg V, Madan AK. Can plants growing in diverse hostile environments provide a vital source of anticancer drugs? J Cancer Ther 2014;10:13-37.

2. Mohanan N, Sivadasan M. Flora of Agasthyamala, Bishen Singh Mahendra Pal Singh. Dehradun; 2002. p. 333.
3. Zhang A, Sun H, Wang X. Recent advances in natural products from plants for treatment of liver diseases. Eur J Med Chem 2013;63:570-57.

4. Han YS, van der Heijden R, Verpoorte R. Biosynthesis of anthraquinone in cell cultures of the Rubiaceae. Plant Cell Tissue Organ Culture 2001;67:201-20.

5. Santos CC, Salvadori MS, Mota VG, Costa LM, de Almeida AA, de Oliveira GA, et al. Antinociceptive and antioxidant activities of phytol in vivo and in-vitro models. Neurosci J 2013;11. http://dx.doi.org/10.1155/2013/949452

6. Gamble JS. Flora of Presidency of Madras. Vol II. Bishen Singh Mahendra Pal Singh, Dehradun; 1921. p. 650-2.

7. Ijinu TP, Anish N, Shiju H, George V, Pushpangadan P. Home gardens for nutritional and primary health security of rural poor of South Kerala. Indian J Traditional Knowledge 2011;10:413-28.

8. Razafimandimbison SG, Bremer B. Nomenclatural changes and taxonomic notes in the tribe Morindeae (Rubiaceae). Adansonia 2011;33:283-309.

9. Sharma MD, Rautela I, Gahlot M, Sharma N, Koshy EP. GC-MS analysis of photo components in juice sample of Indian cane: Saccharum barberi. Int J Pharm Sci Res 2015;6:5147-53.

10. Stein SE. National Institute of Standards and Technology (NIST), Mass Spectral Database and Software. Version 3.02. Gaithersburg, USA; 1990.

11. Mohammad TG, Mohammed HE, Ali J, Seyedhossein H, Mohammad M. Antimicrobial activity, toxicity and stability of phytol as a novel surface disinfectant. Environ Health Eng Manage J 2015;2:13-6.

12. Peter O, Malin H, Lars I Hellgren, Rikard H. Phytol: a chlorophyll component with anti-inflammatory and metabolic properties. Recent Advances in Redox-Active Plant and Microbial Products; 2014. p. 345-59.

13. Netscher T. Synthesis of vitamin E. Vitamins Hormones 2007;76:155-202.

14. Rao CV, Newmark HL, Reddy BS. Chemopreventive effect of squalene on colon cancer. Carcinogens 1998;19:287-97.

15. . Mohan VR, Sudha T, Chidambarampillai S. GC-MS analysis of bioactive components of aerial parts of Kirganelia Reticulata poir (Euphorbiaceae). J Curr Chem Pharm Sci 2013;3:4.

16. Alagammal M, Tresina P, Sand Mohan VR. GC-MS determination of bioactive components of Polygala javana dc. Int J Curr Pharm Res 2012;4:42-4.

\section{How to cite this article}

- Renji R Nair, A Gangaprasad. GC-MS analysis of methanolic stem extract of Gynochthodes ridsdalei, razafim and B. bremer, an endemic, endangered medicinal plant of Southern Western Ghats. Int J Curr Pharm Res 2017;9(3):98-101. 